\title{
HUMAN AND PORCINE VOCALIZATIONS AFTER CREATION OF A HUMAN LARYNX
}

\author{
KARI GREEN*1, ROBERT MORRISON ${ }^{2}$, MATTHEW WHEELER ${ }^{3}$, SCOTT \\ HOLLISTER $^{4}$, and GLENN GREEN ${ }^{*} 1,2$
}

*Corresponding Author: gegreen@med.umich.edu

${ }^{1}$ Kresge Hearing Research Institute, University of Michigan, Ann Arbor, MI, U.S.A.

${ }^{2}$ Department of Otolaryngology - Head and Neck Surgery, University of Michigan, Ann Arbor, MI, U.S.A.

${ }^{3}$ Department of Animal Sciences, University of Illinois at Urbana-Champaign, Champaign, IL, U.S.A.

${ }^{4}$ Department of Biomedical Engineering, Georgia Institute of Technology, Atlanta, GA, U.S.A

Rare medical conditions such as laryngeal agenesis, tracheal agenesis and complete subglottic stenosis physically prevent speech development in cognitively unimpaired children. We identified twelve such children, ranging from 13 months to 7 years of age. Prior to surgical intervention, all of the children had age-appropriate language comprehension despite physical preclusion of laryngeal sound production. To allow these children to develop speech, we have developed novel surgical techniques to construct or reconstruct a larynx and remove the physical obstacles to speech production. These techniques include laryngeal atresia repair (creation of a larynx from surrounding developmental tissues), laryngotracheal reconstruction (using rib cartilage to create a portion of the airway), slide tracheoplasty (excision and reanastomosis to create a patent airway) and laryngeal reinnervation (surgically bringing in another nerve to function for the recurrent laryngeal nerve). For the most complex anatomic defects, we have developed a bioengineered larynx based on typical human anatomy and suitable for surgical implantation - this bioengineered larynx, to date, has only been used in the piglet.

Aphonic children prior to reconstruction had no laryngeal or oral airflow. Although these children had normal hearing and comprehension of auditory input, they were unable to speak. All of their communication to others was via hand gestures, lip and tongue smacking (percussives using articulators without air stream), and manual signs. The communicative abilities of the children were variable; the most proficient child used upwards of 250 ASL signs (Range of all children studied: 0 - 250 signs; Median: 25 signs). After reconstruction, these children had self-controlled laryngeal airflow. Despite already having normal 
language comprehension, these children developed the ability to vocalize via a progression through the same stepwise development of speech that all typical infants experience in acquiring spoken language (Vihman, 1996). Notably, the presence of lip and tongue smacking in these previously aphonic children was not readily coordinated with the now-normal air stream. However, these post-aphonic children progressed to spoken language much more quickly than typical infants. Most surprisingly, the number of signs and modalities used to communicate prior to the surgery did not correlate with speed of speech acquisition after surgery.

As an additional method to allow aphonic children to access speech, we developed a bioengineered human larynx. Fine resolution computed tomography of an adult male human larynx was segmented to create a stereolithographic file of the human larynx with submillimeter accuracy. The file was modified to allow creation of a soft-tissue matrix and the addition of stem cells. Further modification allowed the insertion of a completely-implantable, externallycontrolled sound production source. Larynges were then manufactured in our bioengineering laboratory under well-established conditions for implantable medical devices. Initial testing was in the piglet animal model.

After approval was obtained from the animal use committee, a Yorkshire piglet underwent laryngectomy (excision of its voice box), palatopharyngoplasty (palatal reconstruction into a human configuration) and inferior transposition of the airway. The human bioengineered larynx was then implanted into the piglet in an inferior anatomic position corresponding to adult human laryngeal positioning. Following recovery from anesthesia, the piglet had a human laryngeal configuration with the capacity to produce sounds from an externally controlled Bluetooth device. An iPod was used to control, via Bluetooth, an implanted modified speaker which was a part of the 3D-printed larynx, i.e. all vocalizations were externally controlled by the research team (but not the modification of those vocalizations, equivalent to articulations, which were controlled by the piglet). The distal airway was cannulated for breathing and subglottal pressure was therefore not elevated. Similar to the previously aphonic children after reconstruction, the piglet was able to consistently and repeatedly modify the sounds produced by the human bioengineered larynx with tongue, lip and mouth motion after the reconstruction. The patterns were repetitive and cycling. The repetitive nature was consistent with nonaccidental phonation, similar to the babbling of children, whether developmentally or with reconstructed larynges.

The sine qua non of verbal language is the conscious control of vocal communication. Evolution of spoken language requires transition from involuntary (or vegetative) emotional sound production to conscious sound production; and then from conscious sound production to conscious complex sound production. Bohm et al. (2010) show that after reconstruction, normal human verbal speech development transitions through a series of similar stages: (1) Exclusively vegetative function of the larynx; (2) Voluntary control over vegetative laryngeal functions; (3) Babbling; (4) Simple words; (5) Complex speech. Evolutionary theories of language propose different underlying pathways for the development of complex speech. 
We provide evidence that language comprehension and speech production can evolve separately. During development of speech production in all previously-aphonic reconstructed children, a distinct and prolonged babbling stage was noted. This implies that infants babble as part of a stepwise, normal process to acquire increased laryngeal control (and not due to a lack of language). Moreover, the experience in the piglet demonstrates that nonhuman mammals with human laryngeal positioning and externally-controlled sound production may be able to modify the sounds in nonaccidental patterns. The temporal separation of the acquisition of the children's ability to produce and comprehend language strongly implies that language skills could develop phylogenetically separately from laryngeal control.

Similar to MacNeilage and Davis (2000), we propose that the stepwise acquisition of spoken language, as exemplified in these previously aphonic children, recapitulates the evolution of language.

\section{References}

Bohm, L. A., Nelson, M. E., Driver, L. E., \& Green, G. E. (2010). Babbling, vegetative function, and language development after cricotracheal resection in aphonic children. The Laryngoscope, 120(12), 2494-2497.

MacNeilage P. F. \& Davis, B. L. (2000). Evolution of Speech: The Relation Between Ontogeny and Phylogeny. In Knight, C., Studdert-Kennedy, M., \& Hurford, J. (Eds.). The evolutionary emergence of language: social function and the origins of linguistic form. (pp. 146 - 160) Cambridge, UK: Cambridge University Press.

Vihman M. M. (1996). Phonologic Development: The Origins of Language in the Child. Cambridge, MA: Blackwell Publishers. 